\title{
Asteroids as radial velocity and resolving power standards for medium and high resolution spectroscopy $\star$
}

\author{
T. Zwitter ${ }^{1}$, F. Mignard ${ }^{2}$, and F. Crifo ${ }^{3}$ \\ 1 University of Ljubljana, Faculty of Mathematics and Physics, Department of Physics, Jadranska 19, 1000 Ljubljana, Slovenia \\ e-mail: tomaz.zwitter@funf.uni-Lj.si \\ 2 Observatoire de la Côte d'Azur, Cassiopée, CNRS UMR 5202, BP 4229, 06304 Nice Cedex 4, France \\ 3 Observatoire de Paris, GEPI, 5 place Jules Janssen, 92195 Meudon, France
}

Received 29 June 2005 / Accepted 30 August 2006

ABSTRACT

\begin{abstract}
Echelle spectra of 10 bright asteroids are presented and compared against an observed twilight spectrum and a computed Solar spectrum. Spectra covering a $2130 \AA$ spectral range centered on $\lambda=5785 \AA$ are of high resolving power and high signal to noise ratio. We compare detailed properties of spectral lines and not albedo variations. It is shown that the normalized Solar and asteroid spectra are identical except for radial velocity (RV) shifts which can be predicted at accuracy level of $1 \mathrm{~m} \mathrm{~s}^{-1}$. So asteroids are proposed as new and extremely accurate radial velocity standards. Predicted and measured RVs of observed asteroids match within the limits of accuracy of the instrument. There are numerous absorption lines in the reflected Solar spectrum. This allows a direct mapping of the resolving power of a spectrograph between and along echelle spectral orders. Thus asteroid spectra can be used to test the wavelength calibration and resolving power of spectrographs on the ground as well as in space, including the Gaia mission of ESA. All spectra are given in electronic form.
\end{abstract}

Key words. techniques: radial velocities - techniques: spectroscopic - instrumentation: spectrographs - minor planets, asteroids Sun: photosphere

\section{Introduction}

Spectroscopic observations are usually wavelength calibrated using spectra of standard calibration lamps. Still, it is desirable to check this calibration with real objects on the sky in order to discover possible instrumental effects. These effects include the calibration and the stellar beams not being exactly parallel when they enter the spectrograph slit; uneven illumination of the slit, which causes spectral shifts and therefore errors in the measurement of radial velocity; flexures and/or temperature instabilities (present in most of the spectrographs that are mounted directly on the telescope), which could cause significant variation of velocity and/or spectral shape if not monitored and properly accounted for.

IAU Commission 30 "Radial velocities" has defined a list of standard stars with accurate velocities constant over many years. The list of Elodie-CORAVEL high-precision standard stars (published at obswww.unige.ch/ udry/std/std.html) contains a total of 140 stars, 38 of them with a radial velocity error $\$ 50 \mathrm{~m} \mathrm{~s}^{-1}$ (Udry et al. 1999). Most of these stars are bright $(V<7)$ so they may be unsuitable as radial velocity standards for spectrographs on large telescopes due to saturation problems. The same is true for the now quite numerous stars that are being searched for extrasolar planets. Also, the RV residuals for some of these stars show a peculiar behaviour possibly due to yet undiscovered planetary companions, so their RV is not predictable accurately enough for calibration purposes. What

* Full version of Table 5 is only available in electronic form at the CDS via anonymous ftp to

cdsarc.u-strasbg.fr $(130.79 .128 .5)$ or via

http://cdsweb.u-strasbg.fr/cgi-bin/qcat?J/A+A/462/795 is more, in some cases only the variation but not the absolute value for RV has been derived and published. Nordstrom et al. (2004) published radial velocities of the first $15 \%$ of stars in the Hipparcos catalogue, part of which may be used as references if an error up to $300 \mathrm{~m} \mathrm{~s}^{-1}$ is acceptable. The sample that is being observed by the RAVE survey (Steinmetz et al. 2006) is much larger and fainter, but of moderate accuracy. Most of the stars are observed only once, so some of the radial velocities may belong to (the yet undiscovered) binary stars.

Here we propose to use asteroids as objects for which radial velocity at the time of observation can be easily calculated to an extreme accuracy level. To the best of our knowledge no high resolution spectra of even the brightest asteroids exist in the literature. So we followed our initial suggestion (Zwitter \& Crifo 2003; Mignard 2003) and obtained high resolution and high signal to noise echelle spectra of 10 bright asteroids. Measurements of their radial velocities are compared to the accurate calculated values. Normalized asteroid spectra are also compared to both theoretical and observed (twilight) Solar spectra for each of the echelle orders. We show that asteroid spectra have identical spectral lines to twilight spectra. The excellent distribution of Solar spectral lines over the whole UV, optical and IR domain allows for a more accurate assessment of wavelength accuracy and resolving power than the conventional methods which use telluric lines.

The next section describes the observations and data reduction procedures. In Sect. 3 we briefly describe the computations necessary to derive an accurate prediction of asteroid radial velocity at the time of observation. Observed asteroid and Solar spectra are compared in Sects. 4 and 5 , followed by our conclusions. 
Table 1. Wavelength ranges of individual spectral interference orders of the Asiago echelle spectrograph.

\begin{tabular}{cccc}
\hline \hline Order & Range $[\AA]$ & Order & Range $[\AA]$ \\
\hline 33 & $6720-6850$ & 41 & $5410-5510$ \\
34 & $6520-6650$ & 42 & $5280-5380$ \\
35 & $6330-6460$ & 43 & $5150-5250$ \\
36 & $6160-6270$ & 44 & $5040-5130$ \\
37 & $5990-6110$ & 45 & $4930-5020$ \\
38 & $5830-5940$ & 46 & $4820-4910$ \\
39 & $5680-5790$ & 47 & $4720-4810$ \\
40 & $5540-5650$ & & \\
\hline
\end{tabular}

\section{Observations and data reduction}

Spectroscopic observations were collected with the echelle+CCD spectrograph of the $1.82 \mathrm{~m}$ telescope operated by Osservatorio Astronomico di Padova atop Mt. Ekar (Asiago, Italy). The spectrograph is directly mounted at the Cassegrain F/9 focus of the telescope. The light enters the spectrograph through a $150 \mu \mathrm{m}(1.9$ arcsec $)$ slit which is normally mounted along the $\mathrm{PA}=90^{\circ}$ or $270^{\circ}$ angle, i.e. in the $\mathrm{E}-\mathrm{W}$ direction. The slit length of 12.6 arcsec allows the asteroid to be placed to one side and to collect useful sky background at the other side of the slit length. The position of the object on the slit is monitored by a red sensitive TV guider. The CCD detector is a thinned broad-band coated E2V CCD47-10 with $1 \mathrm{k} \times 1 \mathrm{k}$ square pixels of $13 \mu \mathrm{m}$ and with a quantum efficiency $>70 \%$ in the interval of 3800 to $7500 \AA$. Here we use the 15 echelle orders that cover the 4720-6850 $\AA$ range with small gaps between the orders (see Table 1). Redder orders are littered by telluric absorptions of the Earth atmosphere, while those to the blue have difficulties with accurate determination of the continuum level. The CCD is cooled with liquid nitrogen which renders the dark current negligible. The readout noise is $\sim 9$ electrons. Spectra are wavelength calibrated from spectra of a ThAr lamp which uniformly illuminates the slit. The resolving power of the spectrograph is $\sim 24000$ at central wavelengths of echelle orders, with notable degradation towards the edges (see below) due to optical distortions. Wavelength sampling is from $0.0955 \AA /$ pix in spectral order 47 , to $0.1357 \AA /$ pix in order 33 . The resolution element equals 2.0 pixels.

Observations of 10 bright asteroids were obtained. Table 2 summarizes the observing log. To observe a representative Solar spectrum integrated over the Solar disk we obtained a series of dawn twilight sky exposures. The telescope guiding was switched off during twilight exposures in order to minimize spectrograph flexures and light from chance superposition stars entering the slit. Each asteroid was observed in a series of consecutive exposures with a ThAr calibration lamp spectrum obtained at the start and end of the series. Each spectrum in a series was independently reduced using standard IRAF echelle routines. The routine apall was used to trace and extract the spectrum and to subtract the sky background which was assumed to be the median of pixels at the same wavelength and at the part of the slit not illuminated by the object. The wavelength calibration was performed using both images of calibration lamp spectra. Lamp spectra were extracted with the aperture and tracing information from the object exposure. Wavelength solutions of both lamps were combined using an average weighted by the difference in time at mid exposure between the object and each of the lamp spectra. A few hot stars with very high rotational velocity were observed during each of the observing
Table 2. Log of asteroid observations. $N$ is the number of exposures, $t$ is the total observing time, Date is the truncated date of observation and $V$ is the apparent $V$ magnitude (derived from the Lowell Observatory asteroid database). $S / N$ is the signal to noise ratio per $0.128 \AA$ wavelength bin at $6070 \pm 4 \AA$.

\begin{tabular}{rlrrcrr}
\hline \hline \multicolumn{2}{l}{ Name } & $N$ & $t[\mathrm{~s}]$ & Date & $V$ & $S / N$ \\
\hline 1 & Ceres & 3 & 3000 & 20050403.12 & 7.6 & 188 \\
2 & Pallas & 4 & 3300 & 20050402.95 & 7.3 & 242 \\
3 & Juno & 3 & 3000 & 20040925.81 & 10.4 & 81 \\
4 & Vesta & 4 & 3200 & 20040926.00 & 6.3 & 204 \\
9 & Metis & 3 & 3000 & 20040925.45 & 9.2 & 153 \\
21 & Lutetia & 3 & 3600 & 20040926.13 & 10.7 & 98 \\
27 & Euterpe & 3 & 3600 & 20040927.13 & 10.2 & 214 \\
40 & Harmonia & 3 & 3600 & 20040925.09 & 9.8 & 94 \\
49 & Pales & 3 & 3600 & 20040929.17 & 11.5 & 71 \\
80 & Sappho & 3 & 2700 & 20040928.64 & 11.1 & 84 \\
& dawn sky & 3 & 480 & 20040926.20 & & 340 \\
\hline
\end{tabular}

runs. Among them we used for the final reductions $\lambda$ Eri spectra for the September 2004 observing run and HD 149757 for the April 2005 run. A high order cubic spline fit was used to remove stellar features and thus obtain a normalized telluric spectrum. Each order of observed spectra of asteroids and twilight were normalized using a 5-piece cubic spline fit with $2 \sigma$ (lower) and $3 \sigma$ (upper) rejection limits and with 10 iterations using a growing radius of 1 pixel. The normalized spectra were median combined and the normalized telluric spectrum was divided out to finally obtain the normalized spectrum of a given asteroid or twilight. One of the principal goals of our analysis is to compare a high resolution twilight spectrum to those of asteroids. We note that intensities of individual telluric features can be variable in time, so any spectral range with normalized telluric spectral intensities outside the 0.99-1.01 range was excluded from further analysis. This criterion rejected 488 wavelength bins, so that the final spectra contain normalized intensities at 13665 wavelengths.

Radial velocities were measured against a theoretical spectrum which was calculated using the Kurucz models. We used the latest version of the model of the Solar atmosphere which was kindly provided to us by F. Castelli. The model was computed with the Atlas 9 code using the chemical abundances from Grevesse \& Sauval (1998). Opacity distribution functions were recalculated for these specific abundances (Castelli \& Kurucz 2004). The effective temperature is $5777 \mathrm{~K}, \log g=4.4377$, mixing length scale height is 1.25 and rotational velocity is $2.0 \mathrm{~km} \mathrm{~s}^{-1}$. The overshooting option was not used. The spectrum was calculated with the Linux implementation of the SYNTHE code (Sbordone et al. 2004). The normalized theoretical spectrum calculated at $R=500000$ and degraded to the resolving power of 24000 is reported in Col. 4 of Table 5 .

Measurement of radial velocity of a given asteroid was done for each spectral order separately using the RVSAO package (Kurtz et al. 1992). The values of radial velocities were consistent between the orders, but the bluest 4 orders showed a distinct trend (see below). So the mean of observed velocities in orders 33-43 (with values of the two most deviant orders not taken into account) was used as the final observed radial velocity of an asteroid.

\section{Calculation of radial velocity}

Radial velocity of an asteroid at a given moment can be calculated with extreme accuracy. This is because most asteroids 
have been observed during many $(\sim 30)$ oppositions so that their positions are known to within 1 arcsec for several years in advance. Assuming a typical space motion of $\sim 30 \operatorname{arcsec~}^{-1}$ this corresponds to a time error of only $120 \mathrm{~s}$. The radial velocity of an asteroid changes by $\approx 30 \mathrm{~km} \mathrm{~s}^{-1}$ in 3 months or $0.5 \mathrm{~m} \mathrm{~s}^{-1}$ in $120 \mathrm{~s}$. So we may conclude that radial velocity can be calculated at an $1 \mathrm{~m} \mathrm{~s}^{-1}$ level of accuracy.

The equatorial rotational velocity of an asteroid is of $22 \mathrm{~m} \mathrm{~s}^{-1}$ for an asteroid with a diameter of $250 \mathrm{~km}$ and with a $10 \mathrm{~h}$ rotational period. For the largest and brightest asteroids it is somewhat larger, reaching a maximum of $84 \mathrm{~m} \mathrm{~s}^{-1}$ for Ceres. Most large asteroids are nearly spherical in shape. Also reflectance for most of them (with the exception of Vesta) is close to constant over the whole surface. So the effect of rotation would be a very moderate broadening of the reflected Solar lines. This effect would be very difficult to measure because of the much larger temperature and rotational broadening of intrinsic Solar lines. However during the GAIA mission, which is the main focus of this work, main belt asteroids will not be observed at opposition, but at a phase angle $\sim 20^{\circ}$, and always less than $30^{\circ}$. The gibbous shape of the illuminated surface of such asteroids means that a crescent-shaped part near the limb with possibly high radial velocity is not illuminated. This introduces a small wavelength shift of the integrated reflected spectrum if an asteroid rotates about an axis pointing away from Earth. Assuming a spherical asteroid with a rotation axis in the plane of the sky, the surface of this missing crescent projected on the plane of the sky may reach up to $14 \%$ of the illuminated face at a $30^{\circ}$ phase angle (only $6 \%$ at $20^{\circ}$ ). As the rotational velocity is always smaller than $100 \mathrm{~m} \mathrm{~s}^{-1}$ (see above), this results in a change in the line profile and a Doppler shift smaller than $15 \mathrm{~m} \mathrm{~s}^{-1}$. Notice also that other physical parameters, such as irregularities in shape and reflectance, would also manifest in a radial velocity shift. A hypothetical cigar-shaped asteroid with reflective and black surfaces on the opposite sides from the center of gravity would cause a shift with an amplitude of $1.3 \mathrm{~m} \mathrm{~s}^{-1}$ if its longer axis is $12 \mathrm{~km}$, the rotation period $4 \mathrm{~h}$ and the rotation axis in the plane of the sky. Note that rapid rotation of such an asteroid would be known because of photometric variability. An accurate treatment of asteroid rotation is beyond the scope of this paper. We note however that small radial velocity shifts caused by rotation in principle can be used to disentangle the degeneracy between shape irregularity and reflectance variability in the interpretation of asteroid light curves.

Calculation of a predicted radial velocity of an asteroid at the time of observation includes the following steps:

(a) A photon reaching the Earth at time $t_{2}$ has left the asteroid at the retarded time $t_{1}=t_{2}-r / c$, where $r$ is the distance between the asteroid at the time of photon emission and the observer at its reception. The retarded position is computed by a straightforward iteration of the asteroid orbital motion. The Doppler velocity $R V_{\text {tel }}$ results from the combination of the geocentric radial velocity of the asteroid at time $t_{1}$ and that of the observer due to the rotation of the Earth at time $t_{2}$.

(b) Radial velocity $R V_{\odot}$ of an asteroid to the Sun is determined from its orbit at time $t_{1}$.

(c) The total radial velocity shift of the Solar spectrum reflected by the asteroid and observed by the Earth observer is, for small velocities, $R V=R V_{\odot}+R V_{\text {tel }}$.

We estimate that the values of calculated radial velocities as given in Table 4 are accurate to within $1 \mathrm{~m} \mathrm{~s}^{-1}$. The radial velocity of the Sun which was observed in the scattered light of the dawn sky was calculated similarly. We note that the exact position of scatter is unknown. But since it lies within the $10 \mathrm{~km}$ thick layer of the Earth atmosphere this uncertainty is negligible.

\section{Comparison of calculated to observed velocities}

Table 4 reports calculated radial velocities and compares them to observations. The $\mathrm{O}-\mathrm{C}$ velocity for each echelle order and for each asteroid is plotted in Fig. 1. Note that $\mathrm{O}-\mathrm{C}$ differences are non-random. Each of the observing runs shows a distinctive trend. As already mentioned the telescope slit was kept along $\mathrm{PA}=90^{\circ}$ (September 2004 observing run) and $\mathrm{PA}=270^{\circ}$ (April 2005 run). Non-parallactic angle of the slit meant that, while the stellar image in red wavelengths was kept in the slit by the red-sensitive TV guider, the short wavelength image of the star was partly missing the slit. Asymmetric diffraction of the blue image on the spectrograph slit caused a shift in the angle of the beam entering the spectrograph and led to an appreciable trend of radial velocity error with wavelength. We note that the two runs had the spectrograph mounted at opposite position angles, so Fig. 1 shows opposite trends of velocity shifts. This interpretation is consistent with the fact that the dawn sky spectrum shows no trend of velocity error with wavelength. The reason is that in this case the slit was illuminated uniformly at all wavelengths, i.e. in the same way as for the spectrum of a calibration lamp. As explained in Sect. 2 the final observed radial velocity of an asteroid was calculated without consideration of the bluest spectral orders, so this problem is largely avoided. Note that the observed velocities have errors that are still larger than the shot noise error of $\simeq 0.1 \mathrm{~km} \mathrm{~s}^{-1}$. The reason is spectrograph flexures (Munari \& Lattanzi 1992) that could be corrected only by more frequent calibration lamp exposures.

The mean error in derived radial velocity is $\$ 300 \mathrm{~m} \mathrm{~s}^{-1}$. This is a much smaller value than previous results for this instrument (cf. Siviero et al. 2004). A similar analysis can be performed for any type of spectrograph. The possibility to study radial velocity error as a function of spectral order or position within the order is superior to the usual velocity shift derived from telluric lines, which are almost exclusively confined to the red spectral region. Asteroid spectra are just reflected sunlight, so any unexpected change in spectral properties or radial velocity can be excluded. This is different from standard radial velocity stars for which velocity uncertainties are much larger.

\section{High resolution spectra of asteroids}

Low resolution spectra of asteroids depend on the variation of reflectance with wavelength. But the reflectance stays the same over the small width of spectral lines. Therefore normalized high resolution asteroid spectra are pure reflected sunlight with all the spectral lines of exactly the same shape and depth as in the Solar spectrum. Table $5^{1}$ lists normalized observed spectra of asteroids and twilight together with a theoretical Solar spectrum (see Sect. 2). The presence of material intrinsic to the asteroid, such as regolith, manifests itself in low resolution spectra but is washed away by the normalization in the high resolution case.

This is demonstrated by Fig. 2 where the solid line plots the fraction of wavelength points with $\mathrm{d} y=a b s\left(I_{1}(\lambda)-I_{2}(\lambda)\right) / I_{2}(\lambda)$ below a given value. Here $I$ denotes normalized intensity, and indices 1 and 2 pertain to mean asteroid and twilight spectrum (solid line), theoretical Kurucz and twilight spectrum (long dashes), or twilight and a constant of 1 (short dashes). A relative intensity difference of $3 \%$ occurs in about $7 \%$ of points if we

\footnotetext{
1 Available in electronic form through CDS
} 
Table 3. Radial velocities measured from individual spectral orders. An asteroid's radial velocity was determined as a mean of results for orders 33-43, skipping the 2 most deviant values (see text).

\begin{tabular}{|c|c|c|c|c|c|c|c|c|c|c|c|c|c|c|c|c|}
\hline \multirow{2}{*}{\multicolumn{2}{|c|}{ Name }} & \multicolumn{15}{|c|}{$\overline{\text { Radial velocity from echelle order }}$} \\
\hline & & 33 & 34 & 35 & 36 & 37 & 38 & 39 & 40 & 41 & 42 & 43 & 44 & 45 & 46 & 47 \\
\hline 1 & Ceres & -12.03 & -12.31 & -12.29 & -12.36 & -12.46 & -12.42 & -12.62 & -12.49 & -13.18 & -13.45 & -13.72 & -13.32 & -13.47 & -13.73 & -14.00 \\
\hline 2 & Pallas & 14.14 & 14.18 & 13.96 & 13.78 & 13.47 & 13.71 & 13.46 & 13.68 & 13.04 & 13.19 & 12.82 & 13.64 & 13.64 & 13.67 & 13.18 \\
\hline & Juno & 15.34 & 14.64 & 15.13 & 15.20 & 15.16 & 15.66 & 15.30 & 15.36 & 15.17 & 15.16 & 15.15 & 15.82 & 15.88 & 16.41 & 16.17 \\
\hline 4 & Vesta & 9.73 & 9.69 & 9.95 & 9.89 & 9.82 & 10.14 & 10.28 & 10.56 & 10.23 & 10.24 & 10.36 & 10.84 & 10.79 & 11.12 & 11.19 \\
\hline 9 & Metis & 0.73 & 0.44 & 0.80 & 0.57 & 0.54 & 0.71 & 0.76 & 0.97 & 0.64 & 0.76 & 0.87 & 1.44 & 1.56 & 95 & .77 \\
\hline 21 & Lutetia & -8.24 & -9.15 & -8.66 & -8.86 & -9.09 & -8.26 & -8.63 & -8.79 & -8.67 & -8.60 & -8.70 & -8.11 & -8.27 & -7.90 & -7.98 \\
\hline 27 & Euterpe & -19.54 & -19.80 & -19.69 & -19.78 & -19.93 & -19.38 & -19.55 & -19.47 & -19.77 & -19.59 & -19.68 & -18.95 & -18.78 & -18.51 & -18.65 \\
\hline 40 & Harmonia & -6.50 & -6 & -6.51 & -6.73 & -6.87 & -6.74 & -6.69 & -6.46 & -6.76 & -6.60 & -6.75 & 6 & 05 & & 55 \\
\hline 49 & $\mathrm{P}$ & -13.87 & -13 & -13.43 & -13.76 & -13 & -12.92 & -13.67 & -13.52 & -13.86 & -13.53 & -13 & -13 & -12.66 & -12 & -12.78 \\
\hline 80 & no & -11.0 & -10.54 & -10.46 & -10.76 & -10.76 & -10.18 & -10.48 & -10.33 & -10.73 & -10.59 & -10.57 & -10.04 & -9.78 & -9.48 & -9.61 \\
\hline & twilight & -0.12 & -0.43 & -0.35 & -0.52 & -0.66 & -0.33 & -0.51 & -0.34 & -0.83 & -0.76 & -0.86 & -0.34 & -0.28 & -0.09 & -0.21 \\
\hline
\end{tabular}

Table 4. Calculated radial velocities compared to observations. Columns (3) and (4) give Sun-asteroid and asteroid to Asiago observatory distances in a.u. The next columns are velocities in $\mathrm{km} \mathrm{s}^{-1}$ : Col. (5) is radial velocity of asteroid vs. Sun, Col. (6) is radial velocity of asteroid vs. Asiago observatory and Col. (7) is their sum. Column (8) gives radial velocity as derived from observations (see Table 3).

\begin{tabular}{|c|c|c|c|c|c|c|c|c|c|}
\hline & $\begin{array}{c}\text { Name } \\
\text { (1) }\end{array}$ & $\begin{array}{c}\mathrm{JD} \text { (UTC) } \\
\text { (2) }\end{array}$ & $\begin{array}{l}d_{\odot} \\
\text { (3) }\end{array}$ & $\begin{array}{l}d_{\text {tel. }} \\
\text { (4) }\end{array}$ & $\begin{array}{l}R V_{\odot} \\
(5)\end{array}$ & $\begin{array}{c}R V_{\text {tel }} \\
(6)\end{array}$ & $\begin{array}{c}\text { RV(calc) } \\
\text { (7) }\end{array}$ & $\begin{array}{c}\text { RV(obs) } \\
\text { (8) }\end{array}$ & $\begin{array}{c}\mathrm{O}-\mathrm{C} \\
(9)\end{array}$ \\
\hline 1 & Ceres & 2453463.619167 & 2.66095 & 1.83302 & 1.3092 & -13.9723 & -12.6631 & -12.43 & 0.23 \\
\hline 2 & Pallas & 453463.448085 & 2.38562 & 1.41984 & 3.8052 & 9.6790 & 13.4842 & 13.80 & 0.32 \\
\hline 3 & Juno & |53274.309769 & 2.82011 & 2.50488 & -4.3485 & 19.1563 & 14.8078 & 15.20 & 0.39 \\
\hline 4 & Vesta & 453274.498461 & 2.38032 & 1.41049 & 1.7008 & 8.0025 & 9.7033 & 10.02 & 0.32 \\
\hline 9 & Metis & 453274.449896 & 2.30037 & 1.32308 & -2.3264 & 3.2949 & 0.9685 & 0.70 & -0.27 \\
\hline 21 & Lutetia & 2453274.628148 & 2.17825 & 1.34490 & 2.6739 & -10.9357 & -8.2618 & -8.68 & -0.42 \\
\hline 27 & Euterpe & 2453275.632188 & 2.10125 & 1.28461 & -2.9481 & -16.5471 & -19.4952 & -19.65 & -0.15 \\
\hline 40 & Harmonia & 2453273.592419 & 2.16246 & 1.20745 & 0.1823 & -6.9727 & -6.7904 & -6.66 & 0.13 \\
\hline 49 & Pales & 2453277.665752 & 2.36502 & 1.52997 & -0.3002 & -13.6541 & -13.9543 & -13.60 & 0.35 \\
\hline 80 & Sappho & 2453276.636134 & 1.92316 & 1.26508 & 2.7895 & -13.4288 & -10.6393 & -10.56 & 0.08 \\
\hline
\end{tabular}

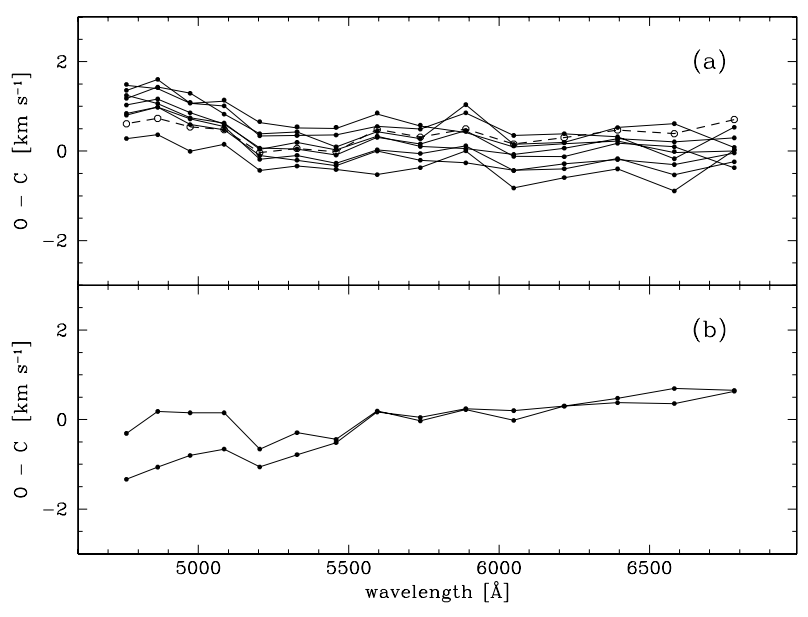

Fig. 1. Difference between observed velocity for the given echelle order and the calculated values. Top panel is for September 2004 observations, the bottom for the April 2005 ones. The twilight spectrum at dawn (dashed line) was observed only in the September 2004 run.

compare the asteroid and twilight spectra, but this fraction increases to about $25 \%$ if we compare observed twilight and theoretically computed spectra. Some $35 \%$ of all points have an intensity at least $3 \%$ fainter than the continuum level. There is no evidence for intrinsic differences between the asteroid and twilight spectrum.

Observations of asteroids are a convenient way to assess the variation of resolving power between and along echelle orders. The Solar spectrum has numerous absorption lines across the



Fig. 2. Cumulative plot of a fraction of wavelength points with two normalized spectra differing by less than a given amount $\mathrm{d} y=a b s\left(I_{1}-\right.$ $\left.I_{2}\right) / I_{2}$. The pairs are mean asteroid and twilight spectrum (solid line), theoretical Kurucz and twilight spectrum (long dashes), and intensities of twilight spectrum compared to a unit intensity (short dashes).

whole UV-IR domain, so it is better suited for this task than the sometimes scarce lines of the calibration lamp spectrum. Also, light from asteroids follows the same path through atmosphere, telescope and the spectrograph as other objects on the sky, so it can be used to assess how the resolving power is influenced by atmosphere variations, accuracy of the telescope focus and of the centering of the object on the slit. Most importantly, such observations can be used to study optical distortions and defocusing along the focal plane which causes a variation in resolving power 
Table 5. Observed spectra of twilight and asteroids rebinned to the same wavelength bins, continuum normalized and Doppler shifted to zero radial velocity. Columns give wavelength (1), twilight (2) and median asteroid intensities (3), normalized flux from the Kurucz Solar model (4), and intensities of individual asteroid spectra (5-14). The complete table is available in electronic form through CDS.

\begin{tabular}{|c|c|c|c|c|c|c|c|c|c|c|c|c|c|}
\hline $\begin{array}{c}\lambda[\AA] \\
(1)\end{array}$ & $\begin{array}{l}\text { Twilight } \\
\text { (2) }\end{array}$ & $\begin{array}{l}\text { Asteroids } \\
\text { (3) }\end{array}$ & $\begin{array}{c}\text { Kurucz } \\
\text { (4) }\end{array}$ & $\begin{array}{l}1 \text { Ceres } \\
\text { (5) }\end{array}$ & $\begin{array}{l}2 \text { Pallas } \\
\text { (6) }\end{array}$ & $\begin{array}{l}\text { 3 Juno } \\
\text { (7) }\end{array}$ & $\begin{array}{l}4 \text { Vesta } \\
(8)\end{array}$ & $\begin{array}{l}9 \text { Metis } \\
\text { (9) }\end{array}$ & $\begin{array}{l}21 \text { Lutetia } \\
\text { (10) }\end{array}$ & $\begin{array}{l}27 \text { Euterpe } \\
\text { (11) }\end{array}$ & $\begin{array}{l}40 \text { Harmonia } \\
\text { (12) }\end{array}$ & $\begin{array}{l}\text { 49 Pales } \\
\text { (13) }\end{array}$ & $\begin{array}{l}80 \text { Sappho } \\
\text { (14) }\end{array}$ \\
\hline 6024.0667 & 0.731 & 0.686 & 0.716 & 0.712 & 0.729 & 0.711 & 0.683 & 0.620 & 0.689 & 0.672 & 0.688 & 0.670 & 0.673 \\
\hline 6024.1951 & 0.821 & 0.797 & 0.839 & 0.794 & 0.814 & 0.822 & 0.791 & 0.766 & 0.809 & 0.802 & 0.800 & 0.783 & 0.774 \\
\hline 6024.3236 & 0.937 & 0.944 & 0.966 & 0.938 & 0.943 & 0.972 & 0.943 & 0.956 & 0.949 & 0.947 & 0.944 & 0.944 & 0.935 \\
\hline 6024.4521 & 0.986 & 0.992 & 0.996 & 0.986 & 0.991 & 1.014 & 0.990 & 0.995 & 0.986 & 1.004 & 0.991 & 1.032 & 0.994 \\
\hline
\end{tabular}



Fig. 3. Resolving power $R \equiv \lambda / \mathrm{d} \lambda$ of the spectrum of 27 Lutetia as a function of wavelength. Points present $R$ of the theoretical Solar spectrum which gave the best match to the observed spectrum in the interval $\pm 10 \AA$ from the point. Points within the given echelle spectral order are connected and the area of the order is shaded.

along and between the spectral orders. An example of such variations is given in Fig. 3 where a normalized theoretical Solar spectrum computed at a resolving power of 500000 has been degraded until it provided the best match for the given $20 \AA$ wide part of the normalized observed spectrum. Variations are partly due to defocusing at the edges of spectral orders. On the other hand, some spectral lines in the theoretical Kurucz spectrum do not match the observed twilight Solar spectrum, as manifested by the long-dashed line in Fig. 2. The procedure therefore tried to compensate for mismatches by adjusting the resolving power, and this causes sharp jumps in Fig. 3. A solution would be to replace the theoretical spectrum with a twilight or asteroid spectrum observed at very high resolving power. Unfortunately no spectra of bright asteroids observed at $R \sim 100000$ and with a high signal to noise ratio exist in the literature.

\section{Conclusions}

Observations of asteroids are convenient to measure radial velocity error as well as resolving power variation along and between the interference orders of an echelle spectrograph. They can be used to assess the accuracy of the absolute wavelength calibration on the level of $\mathrm{m} \mathrm{s}^{-1}$. This is particularly valuable for wavelength calibration of slitless spectrographs, such as the radial velocity spectrograph of the forthcoming Gaia mission of ESA (Katz et al. 2004; Wilkinson et al. 2005). Rotation of asteroids with irregular shape and/or reflectance distribution over their surfaces can introduce radial velocity shifts on the $\mathrm{m} \mathrm{s}^{-1}$ level. Most of the radial velocity standard stars are very bright and could be expected to have a certain degree of intrinsic spectrum variability. Asteroids, on the other hand, are observed at a wide range of magnitudes, allowing a calibration suitable to the saturation limit of the telescope. For the calibration of the GAIA radial velocity spectrometer (RVS) only the brightest asteroids with a fairly round shape will be used.

Asteroid light follows the same path through the atmosphere, telescope and spectrograph as the light from other objects on the sky. The numerous absorption lines in the reflected Solar spectrum therefore allow a detailed mapping of line broadening due to variations in the resolving power along the spectrum. The Solar spectrum is not expected to change much over time, moreover its light output is being constantly monitored. It is therefore appropriate to use the observed width of most individual Solar lines reflected from an asteroid to measure the resolving power of the spectrograph.

Dedicated instruments are used to accurately map the time variability in spectra of small areas on the Solar surface. But spectra reflected from asteroids offer a convenient way to measure the integrated high-resolution spectrum from the whole Solar disk using a normal telescope. In this way a variation of the high resolution integrated Solar spectrum with the Solar cycle could be measured.

Acknowledgements. Generous allocation of observing time with the Asiago telescopes has been vital to this project. We would like to thank Fiorella Castelli, who continues to provide us with insights into the most recent versions of ATLAS and SYNTHE model atmosphere programs, and to Urtzi Jauregi for calculating the theoretical Kurucz spectrum. T.Z. acknowledges fruitful discussions with D. Hestroffer and hospitality of the GEPI group in Meudon. The financial support from the Slovenian Research Agency (to T.Z.) is kindly acknowledged. We thank the referee for very helpful comments.

\section{References}

Beckers, J. M., Bridges, C. A., \& Gilliam, L. B. 1976, A high resolution spectral Atlas of the Solar Irradiance from 380 to 700 nanometers, Vol. 2: graphical form. Sacramento Peak Observatory, Project 7649

Castelli, F., \& Kurucz, R. 2004, in Modelling of Stellar Atmospheres, ed. N. E. Piskunov, W. W. Weiss, \& D. F. Gray

Grevesse, N., \& Sauval, A. J. 1998, Space Sci Rev., 85, 161

Katz, D., et al. 2004, MNRAS, 354, 1223

Kurtz, M. J., et al. 1992, in Astronomical data analysis software and systems, ed. D. M. Worrall, C. Biemesderfer, \& J. Barneseds, ASP Conf. Ser., 25, 432

Mignard, F. 2003, ESA Livelink report

Munari, U., \& Lattanzi, M. 1992, PASP 104, 121

Nordström, et al. 2004, A\&A, 418, 989

Siviero, A., Munari, U., Sordo, R., et al. 2004, A\&A, 417, 1083

Sbordone, L., Bonifacio, P., Castelli, F., \& Kurucz, R. L. 2004, Mem. Soc. Astr. It. Suppl., 5, 93

Steinmetz, M., Zwitter, T., Siebert, A., et al. 2006, AJ, in press [arXiv:astro-ph/0606211]

Udry, S., Mayor, M., \& Queloz, D. 1999, in Precise Radial Velocities, ed. J. B. Hearnshaw, \& C. D. Scarfe, ASP Conf. Ser., 185, 367

Wilkinson, M. I., et al. 2005, MNRAS, 359, 1306

Zwitter, T., \& Crifo, F. 2003, ESA Livelink report, UNILJ/GAIARVS/TN/001.01, accessible on

http://www.mssl.ucl.ac.uk/gaia-rvs/document_list.html 